\title{
Hitting SYK in B-ALL leukaemia
}

Chemotherapy is successfully used to treat some cases of B cell acute lymphoblastic leukaemia (B-ALL), but a proportion of paediatric patients and many adult patients experience treatment failure and/ or relapse. This study showed that spleen tyrosine kinase (SYK) is a target that is active in many types of B-ALL that have a high risk of treatment failure or relapse; inhibition of SYK using a clinically tested inhibitor reduced cancer cell burden in xenotransplantation models.

B-ALL results from the abnormal accumulation of transformed progenitor or precursor B lymphocytes. In their study, the authors investigated the therapeutic potential of targeting SYK because of the key role of this kinase in the activation

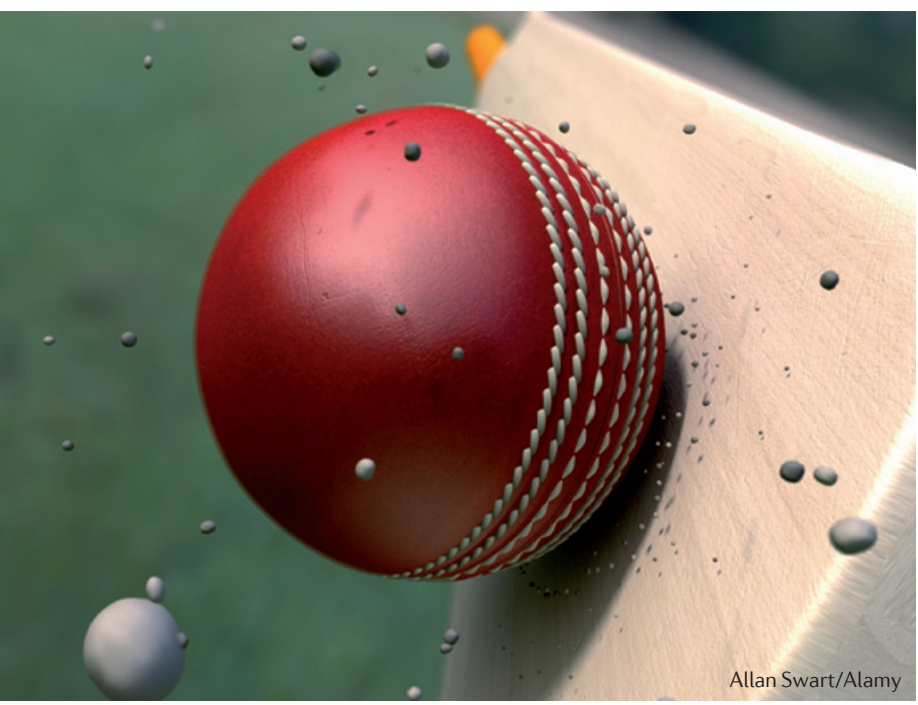

of signalling pathways that regulate the proliferation and differentiation of precursor B cells.

First, the authors used a mouse model of spontaneous B-ALL (Trp53 $3^{--} ; P r k d c^{\text {scid/scid }}$ mice). In B-ALL cells from these mice, SYK was phosphorylated and inhibition of SYK with either fostamatinib (which has been clinically tested in rheumatoid arthritis and B cell lymphoma) or BAY61-3606 (a tool compound) decreased SYK phosphorylation and cell proliferation. Moreover, when leukaemia cells from this mouse model were transplanted into recipient mice (irradiated B6.CD45.1-Rag2 $2^{-/-}$mice), which were then orally treated with fostamatinib, there was reduced cell engraftment compared to non-treated mice. These studies suggest that inhibition of SYK activation reduced the proliferation of B-ALL cells.

Next, the authors used samples from paediatric and adult B-ALL patients with a standard or high risk of relapse and/or treatment failure. In vitro treatment of primary B-ALL cells with the inhibitors reduced the phosphorylation of SYK and inhibited the proliferation of many genetic subtypes of B-ALL cells (including types that did, as well as did not, express the pre-B cell receptor). Further experiments showed that the effects of fostamatinib, which also modulates other tyrosine kinases, were mediated through SYK.

Next, the authors transplanted cells from paediatric B-ALL patients or high-risk adult B-ALL patients into the femur of immunedeficient murine hosts. Initial studies, in which fostamatinib was given immediately after transplantation, showed reduced engraftment of leukaemic cells in at least one type of mouse haematopoietic tissue. Fostamatinib also reduced the burden of well-established leukaemic grafts, which is more clinically relevant, including grafts of several different genetic subtypes. For example, fostamatinib reduced leukaemia burden in all tissues in xenografts from paediatric mixed lineage leukaemia (MLL)rearranged B-ALL, and the drug reduced leukaemia burden in the injected femur and/or other bones in xenografts from many of the remaining types of B-ALL. Moreover, fostamatinib reduced the dissemination of leukaemia cells from bone marrow to other tissues, including the central nervous system; dissemination to this area is a serious complication in B-ALL.

So this study showed that aberrant SYK signalling promotes the growth and survival of diverse genetic subtypes of B-ALL, and that inhibition of SYK may be a therapeutic option for patients who are at high risk of current treatment failure or relapse.

Charlotte Harrison

ORIGINAL RESEARCH PAPER Perova, T. et al. Therapeutic potential of spleen tyrosine kinase inhibition for treating high-risk precursor $\mathrm{B}$ cell acute lymphoblastic leukemia. Sci. Transl. Med. 6, 236 ra62 (2014) 